\title{
MODELO AGROMETEOROLÓGICO DE ESTIMATIVA DE PRODUTIVIDADE PARA O CULTIVAR DE LARANJA VALÊNCIA ${ }^{(1)}$
}

\author{
MARCELO BENTO PAES DE CAMARGO ${ }^{(2,4)}$, ALTINO ALDO ORTOLANI ${ }^{(2)}$, \\ MÁRIO JOSÉ PEDRO JÚNIOR ${ }^{(2)} \&$ SIDNEY MARCOS ROSA ${ }^{(3)}$
}

\begin{abstract}
RESUMO
Interações entre o fator hídrico e a produtividade relativa de laranja 'Valência' (tardia) foram testadas mediante um modelo agrometeorológico matemático. A penalização da produtividade potencial é feita à medida que haja restrição hídrica para a planta durante os estádios fenológicos do pré-florescimento, florescimento e pegamento considerando índices de sensibilidade $(\lambda)$ incorporados numa função multiplicativa. A parametrização e o teste do modelo utilizou dados meteorológicos, fenológicos e de produtividade obtidos da "Fazenda Empreendimentos Agropecuários Cambuhy", localizada no município de Matão (SP) durante os anos agrícolas de 1985/86 a 1996/97. Obtiveram-se os melhores ajustes entre dados observados e estimados com modelo que relaciona o fator hídrico durante os bimestres de agosto/setembro, outubro/novembro e dezembro/janeiro, os quais apresentaram índices de sensibilidade de $-0,08\left(\lambda_{1}\right),+1,18\left(\lambda_{2}\right)$ e $-0,32\left(\lambda_{3}\right)$, respectivamente, para os estádios do préflorescimento, florescimento e pegamento. Como os índices são exponenciais, a magnitude do valor de $\lambda_{2}(+1,18)$ significa que a produtividade da laranjeira 'Valência' é particularmente sensível ao estresse hídrico durante o estádio do florescimento e início do pegamento, correspondente ao bimestre outubro-novembro. A validação do modelo para quatro anos independentes apresentou boas estimativas, com coeficiente de determinação de 0,70 e índice de concordância de 0,87 .
\end{abstract}

Termos de indexação: laranja 'Valência', estádios fenológicos, balanço hídrico, modelo agrometeorológico-matemático, clima-produtividade.

\footnotetext{
(1) Recebido para publicação em 8 de outubro de 1998 e aceito em 17 de fevereiro de 1999 .

(2) Centro de Ecofisiologia e Biofísica, IAC, Caixa Postal 28, 13001-970 Campinas (SP).

(3) Empreendimentos Agropecuários Cambuhy, Matão (SP).

${ }^{(4)}$ Com bolsa de produtividade em pesquisa do CNPq.
} 


\title{
ABSTRACT \\ AGROMETEOROLOGICAL MODEL FOR YIELD PREDICTION OF ORANGE CULTIVAR VALÊNCIA
}

\begin{abstract}
Meteorological data and yields of 'Valência' orange trees from orchards located in Matão (lat.: $21^{\circ} 35^{\prime}$ 'S long.: 48 $25^{\prime} \mathrm{W}$; alt. $551 \mathrm{~m}$ ), State of São Paulo, Brazil, were evaluated with an agrometeorological model. The relative impact of the water stress ratio $(\mathrm{ETa} / \mathrm{ETp})$ was determined for three different stages of growth. The best agreement between observed and estimated data was the ratios that form a multiplicative model for estimating orange yield using field data. These ratios were weighted by applying crop stage dependent exponents or sensitivity coefficients. An analysis of the sensitivity coefficients shows that this model gives higher weight to the water relations during flowering and beginning of the fruit set, which occur in this region in October-November, with $\mathrm{R}^{2}$ higher than 0.70 and d-index of agreement of 0.87 .
\end{abstract}

Index terms: orange, water balance, growth stage, crop yield-modeling, climate-yield relation.

\section{INTRODUÇÃO}

Para determinado local, a combinação copa-cavalo predetermina a produtividade potencial de um pomar de laranja. A expressão dessa potencialidade está associada a outros fatores, a saber: o tipo de solo, a nutrição, a densidade de plantio, as práticas de manejo e, fundamentalmente, o clima. No caso do Planalto Paulista, com dominância de climas tropicais de altitude úmidos e subúmidos, existe um relativo equilíbrio entre a demanda e o atendimento hídrico natural pelas chuvas. Esses parâmetros, analisados por valores médios de balanço hídrico climático, revelam um relativo equilíbrio, com excedentes variáveis no período de verão e déficits hídricos no inverno, distribuídos de forma favorável à fenologia, à produção e à qualidade da fruta (Ortolani et al, 1991). Em condições naturais, sem irrigação, essa distribuição da disponibilidade hídrica expressa a produtividade potencial ou máxima. A análise desse balanço hídrico em períodos menores, semanais ou diários, identifica seqüências de períodos com diferentes intensidades de estresse hídrico que reduzem a produtividade potencial.

A abertura floral é mais favorecida com temperaturas moderadas e umidade relativa baixa (No- gueira, 1979). Para a laranja 'Hamlin', Tubelis \& Salibe (1988) observaram efeito depressivo das chuvas de setembro na produção. Temperaturas muito elevadas, baixo teor de umidade do ar e má disponibilidade hídrica do solo são fatores condicionantes à formação da camada de abscisão na base do pedúnculo das flores, provocando-lhes a queda (Rodriguez, 1991).

$\mathrm{O}$ efeito depressivo da má disponibilidade hídrica no estabelecimento da frutificação foi analisado por Ben Mechlia \& Carrol (1989), em vista da influência do estresse causando por valores elevados da evapotranspiração e da deficiência hídrica durante as fases de florescimento e pegamento dos frutos.

Sequeira (1990) estudou balanços hídricos específicos para citros em cinco localidades paulistas, e determinou a freqüência de decêndios com deficiências hídricas coincidentes com as fases de repouso, do florescimento, do crescimento inicial e do final dos frutos. Para a abertura floral, no fim de setembro, a probabilidade de atendimento hídrico é de $65 \%$ para a região de Limeira e de $55 \%$ para a de Pindorama.

O estudo das interações clima-produtividade é desenvolvido com o uso de modelos que procuram quantificar os efeitos das variações do clima sobre o comportamento vegetal (Robertson, 1983). O estudo do efeito do clima na produtividade vegetal é classi- 
ficado em três tipos de modelos: "empírico-estatístico", "matemático-mecanístico" e "conceitual" (Acock \& Acock, 1991). O "empírico-estatístico", o mais simples, descreve as relações entre as variáveis, sem considerar os processos, apresentando muitas restrições para a extrapolação de resultados. No Brasil, modelos específicos para laranjas foram desenvolvidos por Di Giorgi et al. (1991), Tubelis \& Salibe (1989, 1991). Esses modelos, testados por Camargo et al (1995), apresentaram desempenhos insatisfatórios para locais e material genético diferentes. O modelo "conceitual”, o mais complexo, requer grande número de informações dos processos físicos e biológicos envolvidos, como é o complexo modelo de simulação de crescimento, maturação e produtividade proposto por Ben Mechlia \& Carroll (1989). O “matemático-mecanístico", mais simples que o conceitual, descreve matematicamente a casualidade ou a relação entre os processos envolvidos durante o ciclo da cultura, possuindo menores restrições em relação à extrapolação de resultados do que o empírico-estatístico (Acock \& Acock, 1991).

O trabalho tem por objetivo desenvolver e testar modelo agrometeorológico "matemático-mecanístico" para a estimativa da produtividade ou quebra de produção para a laranja 'Valência', com base na relação entre a produtividade relativa e as condições hídricas ocorridas nos diferentes estádios fenológicos da cultura. O modelo proposto baseia-se na penalização da produtividade segundo as condições hídricas prevalecentes nos estádios fenológicos críticos da cultura. Considera-se que a deficiência hídrica seja o condicionante principal da produtividade da cultura, funcionando como um fator de eficiência, e a produção final seria função da produtividade potencial e da sua interação com as condições hídricas.

\section{MATERIAL E MÉTODOS}

\subsection{Material}

Utilizaram-se dados fenológicos e de produtividade do cultivar Valência, com porta-enxerto limão
'Cravo', da "Fazenda Empreendimentos Agropecuários Cambuhy", localizada no município de Matão, região Norte do Estado de São Paulo (lat.: 21035'S; long.: $48^{\circ} 25^{\prime} \mathrm{W}$; alt. $551 \mathrm{~m}$ ). Os dados de produtividade foram obtidos de quatro quadras, com área aproximada de 5 ha cada uma, de pomares plantados no espaçamento de 7 x 5 m em 1979 e 1980 . O solo predominante é o Podzólico Vermelho-Amarelo, com textura média a argilosa (Prado, 1997). Durante o ciclo da cultura, assinalaram-se épocas do florescimento e da colheita e dados de produtividade por planta (caixas/pé). Os tratos culturais foram semelhantes para todas as quadras, efetuando-se os controles fitossanitários quando necessários.

Para a parametrização dos modelos, consideraram-se oito anos de produção, de 1986 a 1993. A validação foi feita com dados de produtividade independente, relativos a 1994-1997.

Os dados meteorológicos, como chuva e temperaturas máximas e mínimas diárias, foram obtidos no posto agrometeorológico do Instituto Agronômico, localizado na própria Fazenda Cambuhy e próximo das quadras consideradas.

\subsection{Métodos}

\subsubsection{Balanço hídrico}

Para a estimativa da disponibilidade hídrica do solo, empregou-se o modelo do balanço hídrico de Thornthwaite \& Mather (1955) em nível decendial, e o programa desenvolvido por Barbieri et al. (1991), com base nos dados das coordenadas geográficas e de precipitação pluvial e temperatura média do ar, $\mathrm{O}$ modelo estima a disponibilidade hídrica decendial, como deficiência e excedente hídricos, e também a evapotranspiração potencial (EP) e a real (ER).

A ER representa o suprimento de água e, a EP, a necessidade de uma superfície gramada. A evapotranspiração potencial da cultura (EPc) é ajustada pelo coeficiente de cultura $(\mathrm{Kc})$ da forma seguinte:

$$
\mathrm{EPc}=\mathrm{Kc} . \mathrm{EP}
$$

O coeficiente de cultura representa a porcentagem de cobertura do terreno pela parte aérea da cul- 
tura e pelas ervas daninhas (Pereira et al., 1997). Para a cultura adulta de laranja, pode-se considerar uma cobertura de terreno de $80 \%$, ou seja, Kc de 0,80 . Para culturas permanentes, porém, como a laranja, em que as plantas são isoladas e superexpostas à radiação solar e ao vento, admite-se que o valor de Kc seja $20 \%$ superior à cobertura do terreno (Camargo \& Pereira, 1994). Assim, o valor final do Kc seria 0,96. Para simplificação do cálculo dos balanços hídricos, considerou-se o valor de Kc unitário.

O total de água disponível no solo (CAD) foi estimado em função das características físicas do solo da região, que, segundo Reichardt (1985) apresenta umidade na capacidade de campo $(25 \%)$, ponto de murcha (17\%) e densidade global aparente $\left(1,3 \mathrm{~g} . \mathrm{cm}^{-3}\right)$, e para uma profundidade média do sistema radicular de plantas adultas de $100 \mathrm{~cm}$, conforme Shalhevet \& Levy (1990). Considerou-se, assim, nos cálculos dos balanços hídricos, uma CAD máxima de $100 \mathrm{~mm}$.

\subsubsection{Modelo agrometeorológico de estima-} tiva de produtividade

O modelo de penalização proposto por Jensen (1968) e parametrizado por Meyer (1990), Camargo (1993) e Meyer \& Hubbard (1995), para culturas anuais, considera que a produtividade de uma cultura pode ser relacionada às condições hídricas ocorridas durante estádios fenológicos críticos, mediante um modelo multiplicativo:

$$
\frac{\mathrm{Yr}}{\mathrm{Yp}}=\prod_{\mathrm{i}=1}^{\mathrm{n}}\left(\frac{\mathrm{ER}}{\mathrm{EP}}\right)^{\lambda_{\mathrm{i}}}
$$

em que: $Y r=$ produtividade estimada (caixas/pé), Yp = produtividade potencial da laranja 'Valência' (caixas/ pé), Yr/Yp = produtividade relativa, ER/EP = relação entre a evapotranspiração real e a potencial, ao nível decendial; $\lambda=$ índice de sensibilidade e $\mathrm{i}=$ estádio fenológico da cultura.

A razão ER/EP é uma indicação do suprimento hídrico para a planta em relação a sua necessidade (Yao, 1969): aquela inferior à unidade indica que a cultura foi submetida a estresse hídrico.
O modelo baseia-se na penalização da produtividade à medida que haja restrição hídrica para a culura, dependendo do estádio fenológico. A penalização é contabilizada mediante os diferentes índices de sensibilidade. O modelo foi desmembrado para caracterizar três estádios fenológicos críticos da cultura: pré-florescimento, florescimento e pegamento, da seguinte forma:

$$
\frac{\mathrm{Yr}}{\mathrm{Yp}}=\left(\frac{\mathrm{ER}_{1}}{\mathrm{EP}_{1}}\right)^{\lambda_{1}} *\left(\frac{\mathrm{ER}_{2}}{\mathrm{EP}_{2}}\right)^{\lambda_{2}} *\left(\frac{\mathrm{ER}_{3}}{\mathrm{EP}_{3}}\right)^{\lambda_{3}}
$$

em que $E_{1} / E P_{1}, E R_{2} / E P_{2}, E R_{3} / E P_{3}$ e $\lambda_{1}, \lambda_{2}$ e $\lambda_{3}$ são relações entre a evapotranspiração real e potencial e os coeficientes de sensibilidade da cultura durante os estádios de pré-florescimento, florescimento e pegamento respectivamente.

Consideraram-se duas combinações de estádios fenológicos em função dos bimestres, a saber:

Combinação 1: pré-florescimento (jul./ag.), florescimento (set./out.), pegamento-início da frutificação (nov./dez.);

Combinação 2: pré-florescimento (ag./set.), florescimento (out./nov.), pegamento-início da frutificação (dez./jan.).

Os coeficientes de sensibiblidade foram determinados por meio da álgebra matricial, semelhante ao método empregado por Meyer (1990) e Camargo (1993).

\subsubsection{Produtividade potencial}

Em vista da ausência de informações consistentes sobre a produtividade potencial (Yp) do cultivar Valência considerou-se como Yp a maior produtividade obtida durante o período analisado (6,2 caixas/pé), acrescida de $10 \%$, de acordo com o critério sugerido por Kanemasu (1983): Yp = 6,8 caixas/pé.

\subsubsection{Avaliação do desempenho dos modelos}

Para avaliar o desempenho dos modelos na parametrização e nos testes, utilizaram-se as análises de regressão, que fornecem os coeficientes de determina- 
ção $\left(\mathrm{R}^{2}\right)$ e as análises de concordância de Willmot et al. (1985), as quais fornecem o índice d, o erro absoluto médio (EAM), erro sistemático (Es) e erro aleatório (Ea), os quais, quando analisados em conjunto, avaliam com mais consistência o desempenho dos modelos.

\section{RESULTADOS E DISCUSSÃO}

\subsection{Balanços hídricos e produtividades obser- vadas}

Os valores da produtividade relativa observada (Y/Yp) e os valores médios das relações ER/EP de 1985/ 86 a 1996/97, ocorridos nos três estádios fenológicos considerados nas duas combinações encontram-se no Quadro 1. No período 1985/86-1992/93, que serviu de parametrização do modelo, os valores da produtividade relativa observada variaram de 0,47 , em 1986 , a 0,88 , em 1989. A combinação 1 apresentou valores de ER/EP com amplitude de 0,22 a 0,77 para o estádio do préflorescimento (jul./ag.), de 0,22 a 0,99 para o do florescimento (set./out.) e de 0,83 a 1,00 para o do pegamento (nov./dez.). A combinação 2 revelou maior amplitude, de 0,13 a 0,85 , durante o pré-florescimento (ag./set.). Os estádios do florescimento e do pegamento, porém, apresentaram menor amplitude, 0,65 a 0,97 , referente ao florescimento (out./nov.) e de 0,91 a 1,00 para o pegamento (dez./jan.). A menor produtividade relativa $(0,47)$ foi no ano agrícola de $1985 / 86$, coincidente com os menores valores das relações ER/EP observadas nos estádios fenológicos do pré-florescimento e do florescimento, nas duas combinações consideradas. Os anos agrícolas de 1993/94 a 1996/97, que serviram como validação do modelo, apresentaram amplitudes semelhantes das relações ER/EP e da produtividade relativa $(0,51$ a 0,95$)$.

Quadro 1. Relações entre produtividade real e potencial (Y/Yp) e evapotranspiração real e potencial (ER/EP) em função dos anos agrícolas e das combinações de estádios fenológicos considerados

\begin{tabular}{|c|c|c|c|c|c|c|c|}
\hline \multirow{2}{*}{$\begin{array}{c}\text { Ano } \\
\text { agrícola }\end{array}$} & \multirow{2}{*}{$\mathrm{Y} / \mathrm{Yp}$} & \multicolumn{3}{|c|}{ Combinação 1} & \multicolumn{3}{|c|}{ Combinação 2} \\
\hline & & $\begin{array}{l}\mathrm{ER} / \mathrm{EP}_{1} \\
\mathrm{Jul} . / \mathrm{Ag} .\end{array}$ & $\begin{array}{l}\text { ER/EP } \\
\text { Set./Out. }\end{array}$ & $\begin{array}{l}{\mathrm{ER} / \mathrm{EP}_{3}} \\
\text { Nov./Dez. }\end{array}$ & $\begin{array}{l}\text { ER/EP } \\
\text { Ag./Set. }\end{array}$ & $\begin{array}{l}\mathrm{ER} / \mathrm{EP}_{2} \\
\text { Out./Nov. }\end{array}$ & $\begin{array}{l}\text { EREP }_{3} \\
\text { Dez./Jan. }\end{array}$ \\
\hline $1985 / 86$ & 0,47 & 0,30 & 0,22 & 1,00 & 0,18 & 0,65 & 0,98 \\
\hline $1986 / 87$ & 0,61 & 0,71 & 0,78 & 1,00 & 0,79 & 0,90 & 1,00 \\
\hline $1987 / 88$ & 0,59 & 0,58 & 0,49 & 0,95 & 0,47 & 0,71 & 0,99 \\
\hline $1988 / 89$ & 0,88 & 0,22 & 0,47 & 0,90 & 0,13 & 0,90 & 0,91 \\
\hline $1989 / 90$ & 0,71 & 0,71 & 0,90 & 0,85 & 0,85 & 0,86 & 0,92 \\
\hline 1990/91 & 0,67 & 0,77 & 0,74 & 0,89 & 0,78 & 0,76 & 0,93 \\
\hline $1991 / 92$ & 0,86 & 0,47 & 0,56 & 0,83 & 0,25 & 0,80 & 0,98 \\
\hline $1992 / 93$ & 0,77 & 0,54 & 0,99 & 0,97 & 0,74 & 0,97 & 0,99 \\
\hline $1993 / 94$ & 0,72 & 0,78 & 0,94 & 0,90 & 0,85 & 0,90 & 0,99 \\
\hline $1994 / 95$ & 0,59 & 0,46 & 0,25 & 0,89 & 0,21 & 0,63 & 0,90 \\
\hline $1995 / 96$ & 0,95 & 0,49 & 0,69 & 0,93 & 0,29 & 0,99 & 0,94 \\
\hline $1996 / 97$ & 0,51 & 0,39 & 0,87 & 1,00 & 0,66 & 0,91 & 1,00 \\
\hline
\end{tabular}




\subsection{Parametrização do modelo}

Os valores dos índices de sensibilidade, $(\lambda)$, determinados por meio de álgebra matricial, e dos coeficientes de deteminação $\left(\mathrm{R}^{2}\right)$ acham-se no Quadro 2. A combinação 1 apresentou $\mathrm{R}^{2}(0,65)$ inferior ao da 2 $(0,92)$, indicando ser esta a melhor combinação, com índices de sensibilidade de $-0,08,+1,18$ e $-0,32$ para os estádios do pré-florescimento, florescimento e pegamento respectivamente. A magnitude dos índices revela a importância da sensibilidade relativa sobre a produtividade da laranja 'Valência'. Os valores positivos significam que a produtividade é particularmente sensível ao déficit hídrico, especialmente durante o estádio do florescimento $(+1,18)$. Índices de sensibilidade com valor próximo de zero, como no pré-florescimento $(-0,08)$ e pegamento $(-0,32)$, indicam que a produtividade é menos sensível às condições hídricas.

Quadro 2. Valores dos índices de sensibilidade $(\lambda)$ determinados e dos coeficientes de determinação $\left(\mathrm{R}^{2}\right)$ obtidos para as combinações 1 e 2 em função dos estádios fenológicos

\begin{tabular}{|c|c|c|c|c|}
\hline \multirow[b]{2}{*}{$\begin{array}{l}\text { Combi- } \\
\text { nação }\end{array}$} & \multicolumn{4}{|c|}{ Índices de sensibilidade } \\
\hline & $\begin{array}{c}\text { Pré- } \\
\text {-floresc. } \\
\lambda_{1}\end{array}$ & $\begin{array}{c}\text { Flores- } \\
\text { cimento } \\
\lambda_{2}\end{array}$ & $\begin{array}{c}\text { Pega- } \\
\text { mento } \\
\lambda_{3}\end{array}$ & $\mathrm{R}^{2}$ \\
\hline $1 \ldots$. & $-0,39$ & $+0,41$ & $-0,40$ & 0,65 \\
\hline $2 \ldots .$. & $-0,08$ & $+1,18$ & $-0,32$ & 0,92 \\
\hline
\end{tabular}

\subsection{Teste do modelo}

A Figura 1 apresenta as produtividades relativas observadas e estimadas pelas duas combinações para os anos de 1986 a 1993, referentes à parametrização, e para os anos de 1994 a 1997, relativos à validação dos modelos. A combinação 2 apresentou também na validação o melhor desempenho, com boas estimativas das produtividades relativas, sobretudo para os anos agrícolas 1993/94, 1994/95 e 1995/96.

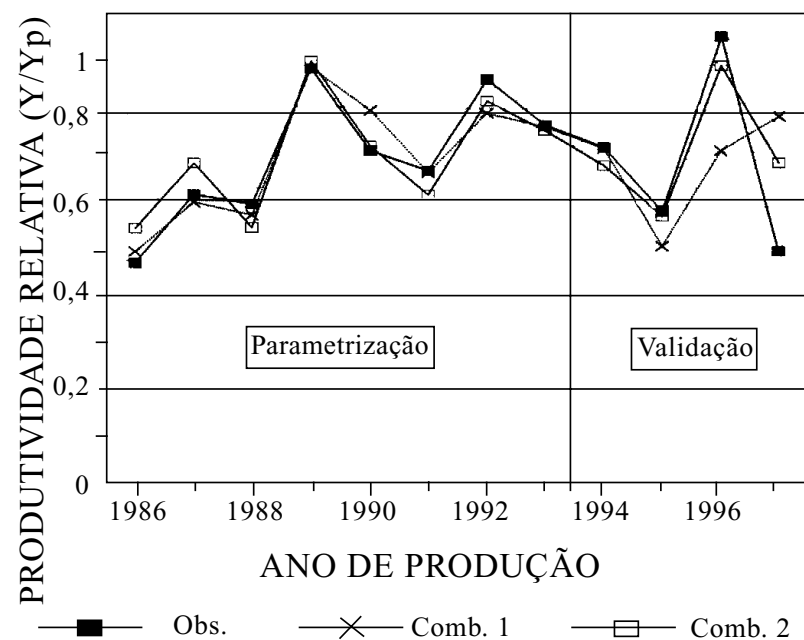

Figura 1. Produtividades relativas (Y/Yp) observadas e estimadas pelas combinações 1 e 2 para os anos agrícolas 1985/1986 e 1996/1997.

O Quadro 3 apresenta os coeficientes de determinação $\left(\mathrm{R}^{2}\right)$, índice de concordância (d), erro absoluto médio (EAM), erro sistemático (Es) e erro aleatório $(\mathrm{Ea})$ do desempenho das duas combinações testadas. A combinação 1 apresentou baixos valores de $\mathrm{R}^{2}(0,41)$ e índice $\mathbf{d}(0,42)$ e, conseqüentemente, altos valores dos erros, em especial do $\operatorname{EAM}(0,15)$ e Es $(0,16)$, indicando estimativas insatisfatórias. $\mathrm{O}$ erro absoluto médio das estimativas em termos de produtividade foi superior a uma caixa/pé.

Quadro 3. Resultados estatísticos da análise de desempenho do teste do modelo agrometeorológico para as combinações 1 e 2

\begin{tabular}{|c|c|c|}
\hline Parâmetro & Combinação 1 & Combinação 2 \\
\hline $\mathrm{R}^{2} \ldots \ldots \ldots \ldots \ldots \ldots$ & 0,41 & 0,70 \\
\hline Índice $d$.............. & 0,42 & 0,87 \\
\hline EAM …................... & 0,15 & 0,07 \\
\hline EAM (cx./pé). & 1,01 & 0,49 \\
\hline Es ......................... & 0,16 & 0,08 \\
\hline Es (cx./pé) ....... & 1,08 & 0,52 \\
\hline $\mathrm{Ea}$ & 0,10 & 0,06 \\
\hline Ea (cx./pé) ...... & 0,69 & 0,43 \\
\hline
\end{tabular}


A combinação 2 revelou valores relativamente altos de $\mathrm{R}^{2}(0,70)$ e de índice $\mathbf{d}(0,87)$ quando comparadas à combinação 1 . Pelos erros de estimativa, observam-se valores reduzidos de EAM $(0,07)$, de Ea $(0,06)$ e de Es $(0,08)$. A combinação 2 resultou em estimativas de produtividade com erro absoluto médio inferiores a 0,5 caixa/pé.

O melhor desempenho das estimativas de produtividade pela combinação 2 baseia-se na penalização da produtividade à medida que haja restrição hídrica para a planta durante agosto/setembro, outubro/novembro e dezembro/janeiro. A magnitude dos índices de sensibilidade revela ser o bimestre outubro/novembro o de maior importância sobre a estimativa da produtividade relativa da laranjeira 'Valência'. Como os coeficientes são exponenciais, o valor positivo de $\lambda_{2}(1,18)$ significa que a produtividade é particularmente sensível ao estresse hídrico durante o estádio fenológico do florescimento.

\section{CONCLUSÕES}

1. O modelo agrometeorológico de estimativa de produtividade da laranja 'Valência', baseado na penalização da produtividade potencial em função da restrição hídrica durante os estádios fenológicos do pré-florescimento, do florescimento e do pegamento apresentou desempenho satisfatório.

2. O modelo que considera o pré-florescimento (agosto/setembro), o florescimento (outubro/novembro) e o pegamento-início de frutificação (dezembro/ janeiro), com índices de sensiblidade $\left(\lambda_{\mathrm{s}}\right)$ de $-0,08$, $+1,18$ e - 0,32 , respectivamente, apresentou os melhores resultados na parametrização e na validação do modelo.

3. A magnitude dos índices de sensibilidade determinados confirmam que o bimestre outubro-novembro, correspondente ao estádio fenológico do florescimento e início do pegamento, é particularmente sensível ao déficit hídrico e, conseqüentemente, o mais importante na estimativa da produtividade relativa da laranja 'Valência'.
4. A validação do modelo agrometeorológico, para quatro anos independentes, apresentou boas estimativas de produtividade, com coeficiente de determinação de 0,70 e índice de concordância (d) de 0,87.

\section{REFERÊNCIAS BIBLIOGRÁFICAS}

ACOCK, B. \& ACOCK, M.C. Potential for using long-term field research data to develop and validate crop simulators. Agronomy Journal, Madison, 83:56-61, 1991.

BARBIERI, V.; TUON, R.L. \& ANGELLOCCI, L.R. Programa de microcomputador do balanço hídrico (Thornthwaite \& Mather, 1955) para dados mensais e decendiais, normais e seqüenciais. In: CONGRESSO BRASILEIRO DE AGROMETEOROLOGIA, 7., Viçosa, 1991. Resumo. Viçosa, 1991. p.297-299.

BEN MECHLIA, N. \& CARROL, J.J. Agroclimatic modeling for simulation of phenology, yield and quality of crop production. I. Citrus response formulation. Int. J. Biometeorology, 33:36-51, 1989.

CAMARGO, A.P. \& PEREIRA, A.R. Agrometeorology of the coffee crop. Geneva, Switzerland, World Meteorological Organizaton, Agricultural Meteorology, 1994. 63p. (CagM Report, 58)

CAMARGO, M.B.P. Determination of the water balance components and drought sensitivity indices for a sorghum crop. Lincoln/USA, 1993. 126p. Doctoral Dissertation, University of Nebraska, 1993.

CAMARGO, M.B.P.; PEDRO JÚNIOR, M.J.; ORTOLANI, A.A. \& ROSA, J.M. Desenvolvimento e teste de modelos agrometeorológicos de estimativa de produtividade de laranjais no Estado de São Paulo. In: CONGRESSO BRASILEIRO DE AGROMETEOROLOGIA, 9., Campina Grande, 1995. Anais. Campina Grande. Sociedade Brasileira de Agrometeorologia, 1995. p.412-414.

DI GIORGI, F.; IDE, B.Y.; DIB, K.; MARCHI, R.J.; TRIBONI, H.R.; WAGNER, R.L. \& ANDRADE, G. Influência climática na produção de laranja. Laranja, Cordeirópolis, 12(1):163-192, 1991.

JENSEN, M.E. Water consumption by agricultural plants. In: KOZLOWSKI,T.T., ed. Water deficits and plant growth. New York, Academic Press, 1968. v.2, p.1-22. 
KANEMASU, E.T. Yield and water-use relationships: some problems of relating grain yield to transpiration. In: TAYLOR, H.M.; JORDAN, W.R. \& SINCLAIR, T.R., eds. Limitations to efficient water use in crop production. Madison, American Society of Agronomy. 1983. cap.9B, p.413-417.

MEYER, S.J. The development of a crop specific drought index for corn. Lincoln/USA, 1990. 165p. Doctoral Dissertation, University of Nebraska, 1990.

MEYER, S.J. \& HUBBARD, K.G. Extending the crop-specific drought index to soybean. In: CONFERENCE ON APPliED Climatology, 9., 1995, Dallas, Texas. Proceedings. Boston, American Meteorological Society, 1995. p.258-259.

NOGUEIRA, D.J.P. O clima na citricultura. In: CITRUS/ Tecnologia de produção. Informe Agropecuário, Belo Horizonte, 52:3-12, 1979.

ORTOLANI, A.A.; PEDRO JÚNIOR, M.J. \& ALFONSI, R.R. Agroclimatologia e o cultivo dos citros. In: RODRIGUEZ, O.; VIÉGAS, R.; POMPEU JUNIOR, J. \& AMARO, A.A., eds. Citricultura Brasileira. 2.ed. Campinas, Fundação Cargill, 1991. v.1, p.153-195.

PEREIRA, A.R.; VILLA NOVA, N.A. \& SEDIYAMA, G.C. Evapo(transpi)ração. FEALQ, Piracicaba, 1997. 183p.

PRADO, H. Os solos do Estado de São Paulo: mapas pedológicos. Piracicaba, Divisão de Biblioteca e Documentação, Campus Luiz de Queiroz/USP, 1997. 205p.

REICHARDT, K. Processos de transferência no sistema soloplanta-atmosfera. 4.ed. Campinas, Fundação Cargill, 1985. 445p.

ROBERTSON, G.W. Guidelines on crop-weather models. Geneve, World Meteorological Organization, 1983. 115p. (World Climate Application Programme, 50)
RODRIGUEZ, O. Aspectos fisiológicos, nutrição e adubação dos citros. In: RODRIGUEZ, O.; VIÉGAS, R.; POMPEU JUNIOR., J. \& AMARO, A.A., eds. Citricultura Brasileira. 2.ed. Campinas, Fundação Cargill, 1991. v.1, p.419475.

SEQUEIRA, E.L.S. Probabilidade de atendimento natural das necessidades hídricas dos citros no Estado de São Paulo. Piracicaba, 1990. 147p. Tese (Mestrado) ESALQ/USP., 1990.

SHALHEVET, J. \& LEVY, Y. Citrus tree. In: STEWART, B.A. \& NIELSEN, D.R., eds. Irrigation of agricultural crops. Madison, American Society of Agronomy, 1990. p.951986. (Agronomy, 30)

THORNTHWAITE, C.W. \& MATHER, J.R. The water balance. Centerton, Drexel Institute of Technology-Laboratory of Climatology, 1955. 104p. (Publications in Climatology, v.8, n. 1)

TUBELIS, A. \& SALIBE, A.A. Relação entre a produtividade de laranjeira 'Hamlin' sobre porta-enxerto de laranjeira 'Caipira' e as precipitações mensais no altiplano de Botucatu. Pesquisa Agropecuária Brasileira, Brasília, 23(3):239-246, 1988.

TUBELIS, A. \& SALIBE, A.A. A estimativa de safra de laranja Hamlin em cinco porta-enxertos. Laranja, Cordeirópolis, 2(10):531-543, 1989.

TUBELIS, A. \& SALIBE, A.A. Efeito da chuva na produtividade da cultura de laranja 'Baianinha'. Laranja, Cordeirópolis, 12(1):141-156, 1991.

WILLMOT, C.J.; ACKLESON, S.G.; DAVIS, J.J.; FEDDEMA, K. \& KLINK, D.R. Statistics for the evaluation and comparison of models. Journal of Geophysical Research, Ottawa, 90(5):8995-9005, 1985.

YAO, A.Y.M. The R index for plant water requirements. Agricultural Meteorology, Amsterdam, 6(4):259-273, 1969. 